\title{
El valor estratégico de los acuerdos de colaboración para la adquisición de conocimiento en innovación abierta Un análisis del sector de las TIC en España
}

\author{
María Guadalupe \\ Calderón Martínez \\ Facultad de Estudios \\ Superiores Cuautitlán, UNAM \\ mgcm.unam@gmail.com
}

\section{Resumen}

La apertura de las fronteras organizacionales para obtener ideas o generar mecanismos alternativos de salida al mercado permite identificar nuevos elementos en la gestión de la innovación. En este trabajo se plantea cómo, a partir de la liberalización del sector de las Tecnologías de la Información y de las Comunicaciones (TIC) en España, las empresas adoptan modelos que motivan la incorporación de conocimientos y agentes externos. La aportación de este artículo es la identificación de los elementos que se relacionan con el establecimiento de acuerdos de colaboración en innovación mediante el análisis de la información contenida en patentes.

Palabras clave: innovación tecnológica, innovación abierta, acuerdos de colaboración, patentes. 


\title{
Strategic value of collaboration agreements for the acquisition of open inno- vation knowledge. An analysis of the ICT sector in Spain
}

\begin{abstract}
Openness in organizations to obtain new ideas or create alternative paths to market, enables the identification of new elements in innovation management. This article sets out how, as of the liberalization of the Information and Communication Technology (ICT) sector in Spain, firms incorporate external agents and expertise. Through the analysis of patent data, this article identifies those elements related to the establishment of collaboration agreements in innovation.
\end{abstract}

Keywords: technological innovation, open innovation, collaboration agreements, patent data.

\section{Introducción}

Una parte central en los procesos de innovación se refiere a la forma de encontrar ideas que se puedan materializar y comercializar. En este contexto, las empresas innovadoras han adoptado estrategias que implican el uso de una amplia gama de actores y fuentes de conocimiento que les permitan alcanzar y mantener sus innovaciones (Schumpeter, 1912, 1942; Rosenberg, 1982; Von Hippel, 1986). El concepto de innovación abierta (Chesbrough, 2003) se define como una estrategia para reorganizar, exteriorizar y mejorar la forma de innovar mediante alianzas de colaboración con otras organizaciones, incluyendo en estos acuerdos, de forma particular, las actividades de I+D+i, la gestión de la propiedad intelectual-industrial, la búsqueda de fuentes externas de conocimiento y los mecanismos alternativos de salida al mercado. El establecimiento de mecanismos de colaboración con otras entidades se considera uno de sus ejes centrales, de ahí que nuestro objetivo sea analizar y describir la adquisición de conocimiento, resultado de una innovación conjunta. Tomando en cuenta este objetivo, planteamos las siguientes hipótesis por contrastar para analizar las variables que explican el establecimiento de acuerdos de colaboración para la adquisición del conocimiento.

H1: El número de inventores que intervienen en los procesos de innovación tiene una relación positiva en el establecimiento de acuerdos de cooperación. 
H2: La incorporación de inventores extranjeros en los procesos de innovación influye en la presencia de acuerdos de cooperación.

H3: El uso de patentes como fuente externa de conocimientos para la innovación influye positivamente en el establecimiento de acuerdos de cooperación.

H4: El uso de artículos científicos como fuente externa de conocimientos para la innovación se relaciona de manera positiva con el establecimiento de acuerdos de cooperación.

En este artículo describiremos primero los antecedentes, conceptos y características sobre los procesos de innovación que nos permitirán dar paso a este modelo. La metodología utilizada se presenta en un anexo donde se detalla la construcción de la base de datos con información obtenida a partir de 345 patentes registradas por empresas españolas del sector de las Tecnologías de la Información y de las Comunicaciones (TIC) durante el periodo de 1999 a 2007. Finalmente, se explica el modelo econométrico que permitirá exponer las conclusiones obtenidas e identificar futuras líneas de investigación.

\section{Marco teórico}

La intensidad del cambio tecnológico, así como el incremento de la complejidad y la incertidumbre en los mercados a causa de un acortamiento en el ciclo de vida de los productos, del incremento en la movilidad de las personas y de la diseminación del conocimiento, han provocado que pocas empresas puedan innovar utilizando sólo sus recursos internos. En este aspecto, lo fundamental son los procesos que permiten generar e intercambiar el conocimiento dentro de las empresas y con otras organizaciones (OECD, 2005b). Así, encontramos la adopción de diversas estrategias que favorecen la colaboración, que van desde la concentración territorial de diversas entidades con características particulares que contribuyen a la creación de un entorno innovador hasta el desarrollo conjunto por iniciativa de las propias organizaciones.

\section{Innovación}

A partir de los trabajos de Schumpeter (1912) se establece la diferencia entre invención, innovación y difusión. Para este autor, una invención es un producto o proceso que ocurre en el ámbito científico-técnico y que perdura en el mismo; la 
innovación es el cambio de índole económico; la difusión, es decir, la transmisión de la innovación, es la que permite que un invento se convierta en un fenómeno económico-social. Asimismo, destaca la decisión del empresario (el innovador) para comercializar un invento, pues es el paso decisivo para conducir a una innovación. La semilla conceptual de Schumpeter fue retomada posteriormente por otros autores como Georgescu-Roegen (1971), Nelson y Winter (1977, 1982), quienes reviven el interés por incorporar al conjunto de herramientas del análisis económico el estudio de la evolución biológica. Cabe destacar la racionalidad limitada de los agentes económicos, dotados de una serie de capacidades, habilidades y conocimientos, que tienen que aprender para adaptarse a su entorno. En todo momento, los agentes deciden en función de sus capacidades y de su entendimiento del mundo, que a su vez depende de sus experiencias, de su aprendizaje y de las capacidades y habilidades del pasado. Del mismo modo, la capacidad de innovar estará influida por el medio que la rodea (Rozga, 1999).

En este contexto se establece la importancia del ambiente en las actividades innovadoras, pues éstas dependen de las herramientas que el medio ofrezca para la toma de decisiones: avances científicos disponibles, soluciones aplicadas en otras empresas, redes de cooperación, etc. Dentro del mismo entorno, la empresa se relaciona con diversas instituciones a través de canales formales e informales (Dosi y Malerba, 1996); por lo tanto, los distintos agentes y sus conexiones influyen en la innovación y su difusión. En la evolución del concepto de innovación, dirigimos la atención hacia la influencia de los factores que dan lugar a la apertura de los procesos de innovación. En este sentido, surge el concepto de Sistema Nacional de Innovación (SNI); es decir, un modelo interactivo de creación y uso del conocimiento en el cual participan los agentes relacionados con la producción y el desarrollo tecnológico (Lundvall, 1992; Nelson 1993; Freeman, 1995 y Metcalfe, 1995). En otras palabras, la innovación y el progreso técnico son resultado de una serie de relaciones entre los agentes que producen, distribuyen y aplican el conocimiento.

\section{Innovación abierta}

No podemos considerar la innovación como un planteamiento endógeno y estático porque la empresa es un espacio social que se relaciona permanentemente con el entorno. Existen situaciones en las que a la empresa le interesa explotar su conocimiento junto con el de las fuentes externas para poder innovar (Morcillo, 2006). En el caso de la cooperación externa, será imprescindible fijar controles de gestión 
para evitar la transmisión de capacidades o habilidades básicas sobre las que descansan las competencias esenciales. El modelo de innovación abierta se concibe explorando y explotando los cambios del entorno, aprovechando la porosidad de las fronteras organizacionales (Chesbrough, 2003); asimismo, hay conceptos que aluden a la vinculación entre agentes de un sistema de innovación, como se observa en el cuadro 1.

\section{Cuadro 1 \\ Modelos abiertos}

\begin{tabular}{|c|c|c|}
\hline \multicolumn{3}{|c|}{ Modelos de carácter estratégico } \\
\hline $\begin{array}{l}\text { Redes de } \\
\text { Colaboración }\end{array}$ & $\begin{array}{l}\text { Saxenian (1994), } \\
\text { Castells (1996), } \\
\text { Shapiro y Varian (1998) }\end{array}$ & $\begin{array}{l}\text { Organizaciones con cierto grado de interdependencia, } \\
\text { que innovan en una gama definida de productos o } \\
\text { servicios. }\end{array}$ \\
\hline $\begin{array}{l}\text { Sistema Nacional de } \\
\text { Innovación }\end{array}$ & $\begin{array}{l}\text { Lundvall (1992), } \\
\text { Freeman (1993), } \\
\text { Metcalfe (1995) }\end{array}$ & $\begin{array}{l}\text { Sistema interactivo de empresas privadas y } \\
\text { públicas, universidades y agencias estatales, para } \\
\text { producir ciencia y tecnología dentro de las fronteras } \\
\text { nacionales. }\end{array}$ \\
\hline Triple Hélice & $\begin{array}{l}\text { Etzkowitz y Leysderdoff } \\
\text { (1995) }\end{array}$ & $\begin{array}{l}\text { Interacción de universidades como productores de } \\
\text { conocimiento, empresas como creadoras de negocios } \\
\text { y administración pública como regulador y generador } \\
\text { de entornos. }\end{array}$ \\
\hline \multicolumn{3}{|c|}{ Modelos de carácter organizativo } \\
\hline $\begin{array}{l}\text { Iniciativas Internas de } \\
\text { Cooperación }\end{array}$ & $\begin{array}{l}\text { Mintzberg (1983), } \\
\text { Handy (1990), } \\
\text { Nonaka y Takeuchi (1995) }\end{array}$ & $\begin{array}{l}\text { Motivación del grupo de proyecto, aprendizaje, } \\
\text { fluidez en la comunicación, clima de confianza dentro } \\
\text { y hacia agentes externos. }\end{array}$ \\
\hline $\begin{array}{l}\text { Iniciativas Externas } \\
\text { de Cooperación }\end{array}$ & $\begin{array}{l}\text { Von Hippel (1986), } \\
\text { Chesbrough (2003), } \\
\text { Leadbeater (2006) }\end{array}$ & $\begin{array}{l}\text { Intercambio de conocimiento, difusión y transferencia } \\
\text { de tecnología. Fuentes externas complementan el } \\
\text { conocimiento interno. Movilización de agentes y } \\
\text { recursos. Dominio de la cartera de competencias } \\
\text { esenciales. }\end{array}$ \\
\hline
\end{tabular}

Fuente: Elaboración propia con información y asesoría proporcionada por el profesor Patricio Morcillo, catedrático de la Universidad Autónoma de Madrid

La innovación abierta constituye una estrategia alternativa a la integración vertical que busca la creación de valor donde algunas empresas necesitan identificar conocimiento externo e incorporarlo, mientras que otras buscan mercados externos para 
sus innovaciones (West y Gallager, 2006). Estudios previos han demostrado que la cooperación entre empresas incrementa el potencial de conocimiento y reduce la duplicidad de esfuerzos (Teece, 1989); además, se sabe que las entidades que participan en diferentes tipos de alianzas son más innovadoras que aquellas que sólo se involucran en una clase de acuerdos por la variedad de conocimiento que se puede obtener a través de esta interacción (Powell, et al., 1999). Los puntos que define este modelo son:

- Se concede la misma importancia al conocimiento interno que al que pueda provenir del exterior.

- Se reconoce el valor de las personas en la incorporación del conocimiento, ya sea que éstas pertenezcan o no a la empresa.

- El objeto principal de este modelo es generar beneficios a través de la $\mathrm{I}+\mathrm{D}+\mathrm{i}$.

- Se propicia la existencia de flujos tecnológicos y de conocimiento.

- Existe un incremento en el número de intermediarios en los procesos de innovación.

- Aparecen nuevos indicadores para medir la capacidad de innovación

- Se favorece el uso de la propiedad intelectual-industrial como fuente de información y su adquisición.

Los análisis empíricos en el tema se encuentran en una etapa inicial, por ello, en este artículo deseamos exponer los resultados obtenidos para la propuesta de un modelo que permita explicar algunos de los aspectos que conforman el modelo de innovación abierta.

\section{Elementos de análisis del modelo}

La literatura establece que si se toman en cuenta los costos que implica la investigación, la amplitud en la distribución del conocimiento y el grado en el avance científico-tecnológico la mayor parte de las innovaciones se lleva a cabo en el seno de empresas, universidades y organismos públicos de investigación (OPI). Es muy difícil que exista el inventor que solo genere una idea singular y la plasme en una innovación (Jacquemin et al. 1986; Niosi, 1993; González y de la Sota, 2006). Por lo tanto, nos interesa analizar si existe una relación entre el número de inventores y el establecimiento de acuerdos de colaboración. Los vínculos entre éstos tienen efectos en dos sentidos al aparecer interrelacionados en una patente. Una 
vez que se concreta un acuerdo de colaboración y se genera una invención no sólo se protege la propiedad intelectual del titular y los inventores, sino que se genera información y una fuente de inspiración valiosa para investigadores e inventores (WIPO, 2005).

Los acuerdos de colaboración, con la participación de inventores extranjeros, tienen conexión con otros factores. En primer lugar, la posición de España como un innovador moderado sobre todo en sectores de alta tecnología (Innometrics, 2009) lleva a la búsqueda de alianzas para obtener conocimientos externos cuando la complejidad tecnológica se incrementa (Guellec y Van Pottelsberghe, 2000). En segundo, desde otro punto de vista, se podrían analizar las motivaciones para la cooperación, distinguiendo entre los beneficios directos a la investigación y los beneficios indirectos estratégicos, económicos o políticos. Existen estudios que abordan la cooperación en investigación desde distintos ángulos, el fenómeno emergente de la cooperación entre los países industrializados se manifiesta en un notable incremento en distintos ámbitos de la cooperación científica en Europa (Aho et al., 2006). Otro estudio trata sobre las estructuras e instrumentos en los sistemas europeos de transferencia de tecnología más dinámicos (Rubiralta, 2004); en él se hace énfasis en el papel de la colaboración entre los grupos de inventores, principalmente desde universidades y centros tecnológicos con dirección hacia las empresas en áreas geográficas destacadas. Por lo anterior, nuestra segunda hipótesis se refiere a la influencia de la incorporación de inventores extranjeros.

La hipótesis sobre el uso de otras patentes como fuente externa de conocimientos para la innovación se basa en las aportaciones de Jaffe et al. (1993) y Verspagen (2004), donde se menciona que las citas de patentes han sido típicamente utilizadas en la literatura para analizar la medida en que el conocimiento es transferido entre individuos, organizaciones y ubicación geográfica. Éstas, en combinación con la presencia de un acuerdo de colaboración, proporcionan información original y directa sobre las fuentes de las derramas de conocimiento ya sea que se generen dentro de la empresa, o si se accede a ellos por medio de fuentes externas (European Commission, 2005). Por otro lado, al estudiar un sector de alta tecnología, se considera que el aumento en la complejidad de las innovaciones implica la incorporación de fuentes de conocimiento externas a la empresa (Guellec y Van Pottelsberghe, 2000). La última hipótesis pone de relieve las relaciones cienciatecnología en procesos abiertos (Fabrizio, 2006); es decir, cómo se incorpora el conocimiento científico por medio de lo que se conoce como citas a documentos no-patente que aparecen en el informe sobre el estado de la técnica. Con respecto 
a este último conjunto, su análisis nos permite obtener información acerca de autores e investigadores, instituciones, revistas citadas, cooperación, carácter básico o aplicado de la investigación, las temáticas y la proximidad entre la patente y la literatura citada (Plaza y Albert, 2004). Con estos argumentos se considera necesario realizar una investigación que permita valorar el modelo y que pueda tener una contribución empírica a la disciplina y a los actores involucrados como son la sociedad y la empresa.

\section{Uso de los datos de patentes}

La idea de utilizar los datos de patentes, en la investigación en ciencias económicas, empieza con Schmookler (1966), seguido por Scherer (1982) y Griliches $(1984,1990)$. Las patentes contienen una riqueza de información sobre la invención patentada, incluyendo datos sobre la identidad y ubicación de los inventores, así como de su institución de procedencia. También contienen citas a patentes anteriores, el análisis de estas citas abre la posibilidad de detectar los enlaces entre invenciones. De esta forma, las patentes se reconocen como una fuente de datos importante y fructífera para establecer vínculos entre invenciones, inventores, científicos, empresas, países, etc. Los datos de patentes incluyen información acerca de las citas a otras patentes y a la literatura científica — no patentes—; además del recuento, las citas de patentes permiten el estudio de los flujos de conocimiento (spillovers), así como la construcción de otros indicadores para medir el impacto tecnológico (Hall et al. 2002; Jaffe, 1986).

Después de haber detectado que las citas parecen estar relacionadas con el valor de las innovaciones (Trajtenberg, 1990), llevamos a cabo un análisis para demostrar su utilidad como indicadores de los flujos del conocimiento en innovación abierta (Caballero y Jaffe, 1993; Jaffe, et al. 1993). Además de las citas de patentes, utilizamos la información acerca de investigadores y solicitantes, como indicador del establecimiento de redes de colaboración (Maspons y Escorsa, 2004).

Con relación a los datos de patentes, éstos pueden ser analizados y agregados de varias maneras, inclusive se pueden combinar según el objetivo de las necesidades de investigación abordando distintos enfoques siempre con prudencia en la utilización e interpretación de los resultados (Hidalgo, 2003; OECD, 1994). El uso de patentes en la investigación económica en ocasiones se ve dificultado por el hecho de que varían ampliamente los conceptos de cómo utilizar esta fuente de informa- 
ción. Cabe destacar que el simple conteo de patentes no constituye un indicador de innovación (Maspons y Escorsa, 2004; Trajtenberg, 1990); sin embargo, el propósito de este artículo es presentar la patente como fuente de datos que permita identificar la presencia de elementos externos en la adquisición de conocimiento en el contexto de la innovación en el sector de las Tic en España. De esta forma, mediante el análisis empírico de los datos seleccionados, superamos las limitaciones del recuento simple.

\section{Contexto del sector de las TIC en España}

La industria de las TIC en España engloba los sectores de electrónica de consumo, servicios de telecomunicación e Internet, equipos de telecomunicación, servicios audiovisuales, contenidos digitales y productos audiovisuales, hardware informático, software informático y servicios informáticos. La creciente integración de los mercados lleva hacia un crecimiento de la parte intangible de la actividad económica con la presencia de flujos de conocimiento, imprescindibles para dar respuesta a mayores complejidades. Resultado del proceso de innovación, las TIC aumentan y transforman la creación y la aplicación económica del conocimiento (Villaseca y Torrent, 2008). Sin embargo, el sector de las TIC requiere una introducción periódica de ideas que circulen en las redes de conocimiento, que permitan soportar una oferta atractiva de nuevos y mejores servicios, que con su utilización posibiliten la creación de nuevos hábitos que deriven en necesidades más sofisticadas en el mercado.

Existen diversos trabajos que han descrito las principales tendencias en el uso de los acuerdos de colaboración por parte de empresas españolas (García, 1992; Cassiman, 1999; Bayona, 2001; Álvarez et al. 2005; Arranz et al. 2008; López, 2008). Partiendo de datos construidos con diversas metodologías, en general se observa una tendencia hacia el predominio de los acuerdos internacionales, el mayor número de ellos es con empresas europeas; además, se observa que existe un elevado peso de los acuerdos suscritos por dos socios. En el sector de las TIC, en la industria española la temática es muy diversa y se inclina hacia la sociedad de la información o al uso de las TIC como medio para mejorar la colaboración (COTEC, 2000; Villaseca y Torrent, 2008; Asimelec, 2009). Los estudios son más escasos $\mathrm{y}$ recientes, pero no hemos encontrado fuentes precisas a las que podamos hacer referencia donde se analice la cooperación en el sector, podemos citar a Rojo y Gómez (2006) que analizan la producción científica y tecnológica de la industria 
española en el sector de las TIC. El aporte de ese artículo es enriquecedor porque estudia el vínculo ciencia-tecnología en empresas del sector a través de las citas en patentes; por ello, contribuye como fuente de datos y apoyo metodológico. Debido a que no hay literatura sobre la colaboración en empresas del sector TIC en España, nos llevó a la búsqueda de datos e información adicional, por lo tanto, se propone desarrollar la parte empírica con la información obtenida en patentes.

\section{Estimación del modelo}

Si bien en la revisión de la literatura hemos visto que la innovación abierta conforma un modelo definido por la interacción de un gran número de variables, nuestro trabajo se concentra en el análisis de la influencia de aquellos factores relacionados con el uso de fuentes externas de conocimiento y las características de los participantes en el establecimiento de los acuerdos de colaboración. El modelo que se propone con base en la revisión de la literatura, y en el análisis descriptivo de los datos obtenidos, quedaría definido por la relación que sobre la Cooperación para la Innovación (CoopInnov), como variable dependiente, tienen la Influencia de los Participantes (PrtInf) determinada por las variables INVNUM e INVDAT y las Fuentes Externas de Conocimiento (ExtSrc) construida a partir de PATDAT y CITDAT, como variables explicativas, tal como se detalla en la metodología de la investigación.

Al utilizar CoopInnov como variable dependiente en el modelo, se pretende dar cuenta de la estrategia para adquirir el conocimiento que se encuentra fuera de las fronteras organizacionales y cómo apropiarse de los beneficios de las actividades innovadoras a través de un modelo de negocios (Teece, 1986; Chesbrough, 2003 y 2006); es por esto que en este punto se desea resaltar el uso de mecanismos formales que permiten a las organizaciones el intercambio de conocimientos con su entorno. El haber determinado la variable dependiente CoopInnov lleva a enumerar las tendencias en este tipo de acuerdos en los datos obtenidos y que resultan fundamentales para nuestra investigación. Existe un peso distribuido uniformemente entre los socios elegidos al momento de establecer el acuerdo de colaboración. Ésta es una tendencia que se aprecia en la práctica cuando en la cooperación en I+D hay una mayor propensión a colaborar con universidades cuando se requiere obtener conocimientos que en otras áreas funcionales, distintas a las competencias esenciales que se poseen (Jacquemin et al. 1986; Niosi 1993; Hagedoorn 1993). Aunque la mayor parte de acuerdos de cooperación en I+D se componen por la participación de dos socios, el peso en este tipo de acuerdos oscila en torno al $60 \%$ 
(Jacquemin et al. 1986, Niosi 1993), mientras que en acuerdos de otro tipo, el peso de los acuerdos de dos socios oscila en torno al 80\% (García, 1995). Los sectores con mayor intensidad tecnológica, es decir, los de mayor gasto en I+D - como es el caso del sector de las TIC - son los que mayor número de acuerdos de I+D tienen (Hagedoorn 1993). Esto pone de manifiesto que la decisión de cooperar en este ámbito no sólo obedece a la falta de recursos financieros.

El análisis de los datos extraídos de la base de datos de patentes se presenta en el cuadro 2. Al conjunto de variables dicotómicas (en escala nominal) que hemos relacionado con este fenómeno, y que se mencionan a continuación, se les ha asignado el valor 1 cuando cumplen con el atributo que deseamos encontrar y 0 cuando éste no se presenta. Por otro lado, respecto a la nacionalidad de los inventores (INVNAT) la media nos indica que un 19\% de ellos son extranjeros; en cuanto a la variable PATDAT, ésta indica que en el $75 \%$ de los casos podemos encontrar citas de otras patentes en el informe sobre el estado de la técnica.Analizando las citas de documentos no-patente, es decir, las citas de artículos científicos (CTIDAT), se puede observar que la media indica que el 17 por ciento de las patentes de la población estudiada incluye este tipo de elementos.

\section{Cuadro 2}

Estadísticos descriptivos de las variables analizadas

\begin{tabular}{lccccc}
\hline & CoopInnov & INVNUM & INVNAT & PATDAT & CITDAT \\
\hline $\mathrm{N} \quad$ Válidos & 345 & 345 & 345 & 345 & 345 \\
$\mathrm{~N} \quad$ Perdidos & 0 & 0 & 0 & 0 & 0 \\
Media &, 41 & 3,14 &, 19 &, 75 &, 17 \\
Desv. Típ. &, 492 & 2,185 &, 389 &, 435 &, 372 \\
Mínimo & 0 & 1 & 0 & 0 & 0 \\
Máximo & 1 & 12 & 1 & 1 & 1 \\
\hline
\end{tabular}

El resto de elementos por estudiar corresponden a datos cuantitativos (en escala proporción). En la variable que contiene la información sobre el número de inventores (INVNUM), el mínimo detectado es de un individuo con un máximo de doce y un promedio de dos inventores por patente. Es pertinente resaltar, basándonos hasta ahora en datos descriptivos, que este promedio indica que no se recurre con frecuencia a la figura del inventor en solitario; sin embargo, en este momento, no podemos relacionar este resultado con el posible establecimiento de un acuerdo de cooperación. 
Para cumplir los objetivos del presente artículo (identificar las variables que con un cierto grado de significación estadística explican la adquisición de conocimientos por medio del establecimiento de acuerdos de cooperación para la innovación) se utilizará una regresión logística binomial. El uso de dicho análisis econométrico es adecuado cuando la variable dependiente utilizada es binaria (Gujarati, 2006). En los resultados que se muestran a continuación es posible observar tanto los coeficientes beta de las variables independientes, su grado de significación y la significación global del modelo. Para este análisis utilizamos el programa SPSS versión 15, con una regresión logística binomial, y empleamos el método por pasos hacia adelante; la estimación finaliza en el número de iteración cuatro, incluyendo todas las variables explicativas introducidas que se ajustan al modelo.

\section{Cuadro 3}

Tabla de clasificación a

\begin{tabular}{|c|c|c|c|c|c|}
\hline & \multicolumn{2}{|l|}{ Observado } & \multicolumn{3}{|c|}{ Pronosticado } \\
\hline & & & \multicolumn{2}{|c|}{ Acuerdo de Cooperación } & \multirow{2}{*}{$\begin{array}{c}\text { Porcentaje correcto } \\
\text { NO }\end{array}$} \\
\hline & & & NO & Sí & \\
\hline \multirow[t]{3}{*}{ Paso 1} & Acuerdo de Cooperación & NO & 164 & 41 & 80,0 \\
\hline & & Sí & 58 & 82 & 58,6 \\
\hline & Porcentaje global & & & & 71,3 \\
\hline \multirow[t]{3}{*}{ Paso 2} & Acuerdo de Cooperación & NO & 176 & 29 & 85,9 \\
\hline & & Sí & 59 & 81 & 57,9 \\
\hline & Porcentaje global & & & & 74,5 \\
\hline \multirow[t]{3}{*}{ Paso 3} & Acuerdo de Cooperación & NO & 184 & 21 & 89,8 \\
\hline & & Sí & 59 & 81 & 57,9 \\
\hline & Porcentaje global & & & & 76,8 \\
\hline \multirow[t]{3}{*}{ Paso 4} & Acuerdo de Cooperación & NO & 178 & 27 & 86,8 \\
\hline & & Sí & 46 & 94 & 67,1 \\
\hline & Porcentaje global & & & & 78,8 \\
\hline
\end{tabular}

a El valor de corte es ,500

En síntesis podemos comentar que la significatividad global del modelo es < a 0,05 por lo cual se considera representativo para el fenómeno estudiado y en cada uno de los pasos la variación de Chi-cuadrado aumenta considerablemente. Para valorar la calidad del ajuste obtenido es preciso recordar que el objetivo de la regresión es explicar los cambios que se dan en la variable dependiente. Si bien un coeficiente $\mathrm{R}^{2}$ tiene un valor absoluto que oscila entre el 0 y el 1 , sabemos de las limitaciones de nuestro análisis, que en nuestro caso el $\mathrm{R}^{2}$ es de 0,522 . Sin embargo, los resul- 
tados de la regresión pueden ser auspiciosos para la realización de interpretaciones iniciales sobre el comportamiento de las variables y del cumplimiento o no de las hipótesis. Los resultados de la prueba de Hosmer y Lemeshow, para evaluar la bondad de ajuste del modelo, muestran que la significatividad es superior al 0,05 por lo que el modelo ajusta nuestros datos. En la tabla de clasificación se han obtenido resultados adecuados que predicen en un $79 \%$ los resultados pronosticados (cuadro 3). Se observa también un número bajo de fallos al momento de que las variables explicativas definen la presencia o no de la colaboración.

A partir de los resultados obtenidos en la regresión por el nivel de significación podemos concluir que las variables propuestas pueden ser incluidas en el modelo (cuadro 4).

\section{Cuadro 4}

Variables en la ecuación

\begin{tabular}{ccccccc}
\hline Variable & B & E.T. & Wald & Gl & Sig. & Exp(B) \\
\hline INVNUM &, 307 &, 077 & 15,854 & 1 &, 000 & 1,359 \\
INVNAT & 2,296 &, 450 & 26,075 & 1 &, 000 & 9,930 \\
PATDAT & 2,454 &, 532 & 21,241 & 1 &, 000 & 11,630 \\
CITDAT & 1,609 &, 384 & 17,551 & 1 &, 000 & 4,997 \\
Constante & $-4,108$ &, 577 & 50,776 & 1 &, 000 &, 016 \\
\hline
\end{tabular}

De igual forma, al observar la matriz de correlaciones, se aprecia que éstas no son altas en las variables que justifican el modelo; además, según las estimaciones condicionales de los parámetros de verosimilitud y su grado de significatividad, se confirma la introducción de éstas, por lo tanto, el modelo final obtenido se expresaría de la siguiente forma:

$$
\log \frac{P}{1-p}=-4,108+0,307 I N V N U M+2,296 I N V N A T+2,454 P A T D A T+1,609 C I T D A T
$$

Por medio del análisis de las variables incluidas en el modelo, detectamos el establecimiento de acuerdos de cooperación cuando aparece más de un titular en las patentes. Por otro lado, la incorporación de los inventores muestra el grado de complejidad y el aporte de conocimientos que cada uno de éstos hace a la actividad inventiva. Este dato es combinable con las citas de patentes para determinar el grado de novedad de la innovación, pues si aparece un número limitado de inventores y un gran número de citas puede significar que la empresa está basando la innovación en otras patentes más que en el desarrollo propio, porque una elevada 
cantidad de citas y un reducido número de inventores pone en duda la novedad, disminuyendo la complejidad de la tecnología. Estos aspectos sugieren otras líneas de investigación en el tema de innovación abierta y sobre el uso de patentes en el análisis económico.

\section{Conclusiones y futuras líneas de investigación}

Con las variables incluidas en el modelo, podemos establecer que tanto el número de inventores INVNUM y su nacionalidad INVNAT, así como las citas de patentes PATDAT y las citas de artículos científicos CITDAT, influyen de manera positiva en los mecanismos de colaboración, con lo cual podríamos confirmar nuestras cuatro hipótesis.

La primera hipótesis acerca del número de inventores que interviene en los procesos de innovación, así como la segunda hipótesis que se refiere a la incorporación de inventores extranjeros representan el incremento de la cooperación tecnológica. Las variables relacionadas con el número y la nacionalidad de los inventores enfatizan aspectos como la movilidad de las personas para el aprendizaje, producción y difusión del conocimiento. Asimismo, se aprecia la necesidad de construir indicadores utilizando información de los inventores, relacionándola con el país, sector industrial, sector público o privado, otros inventores, patentes en el extranjero, etcétera. La tercera hipótesis establece que el uso de patentes como fuente externa de conocimientos influye positivamente en el establecimiento de acuerdos de cooperación; esto se confirma por los aportes de conocimiento codificado, pues describe tecnologías, procedimientos o productos orientados a aplicaciones concretas; su utilidad fue expuesta en el marco teórico. Con relación al empleo de artículos científicos, su aporte al modelo es poco significativo, pero en el último paso se agrega al modelo. Una de las posibles causas de la baja aparición de este tipo de citas puede atribuirse a las deficientes relaciones entre el ámbito científico y el sector industrial, o la ausencia de relaciones directas entre las empresas que desarrollan la tecnología y las universidades que generan conocimiento científico.

Ambos conjuntos de variables, es decir, la Influencia de los Participantes (PrtInf) y las Fuentes Externas de Conocimiento (ExtSrc), explican el fenómeno de estudio, por lo que debemos destacar que estos factores tienen un efecto positivo en la variable dependiente. De forma complementaria a la interpretación de los resultados, podemos afirmar que tal parece que las organizaciones han comprendido que una 
forma de ser competitivas en áreas donde el dinamismo tecnológico es muy rápido o complejo consiste en ser capaces de identificar los conocimientos requeridos en cualquier lugar donde existan.

De la misma manera las autoridades públicas han sido conscientes de este cambio y han tratado de adaptar los programas e instrumentos promoviendo el fortalecimiento de universidades y empresas nacionales. El Programa Marco de Investigación y Desarrollo Tecnológico de la Unión Europea y el Plan Nacional español de I+D+i constituyen ejemplos de esta tendencia (León, 2007). Si bien en los datos se aprecia que la colaboración se refuerza en el periodo comprendido por nuestro análisis, también se muestra que en este sector el número de agentes que cooperan sigue siendo inferior a los que no cooperan. Tal vez estos datos son consecuencia de una debilidad en el sector industrial español, de la escasez de contactos permanentes con grupos de investigación o del desajuste de intereses potenciales.

Otro de los elementos fundamentales es la obtención de conocimientos a partir de fuentes externas a la organización y la forma en cómo acceder a éste para su correcta explotación. Sin embargo, una de las debilidades del sistema español es el escaso nivel de cooperación entre los actores del sistema y, de manera especial, entre el sistema público y privado para ejecutar actuaciones de I+D e innovación (OECD, 2006). La información examinada revela que la industria española se sustenta en el conocimiento científico para desarrollar innovaciones potenciales en una proporción muy inferior al conocimiento tecnológico derivado de la tecnología patentada por la propia industria. Estos resultados originan varias líneas de reflexión sobre el diseño de las políticas científicas y tecnológicas nacionales y regionales, principalmente para aquellas encaminadas a estimular la cooperación entre el ámbito científico y tecnológico, para las políticas que favorezcan el desarrollo de la ciencia doméstica en campos científicos relacionados con sectores de cierta especialización regional o políticas de difusión que favorezcan el acceso de los sectores tecnológicos a la ciencia. En cualquier caso, debe tenerse en cuenta que la interpretación de los flujos ciencia-tecnología efectuada en este trabajo se restringe al conocimiento codificado implícito en los datos recogidos en las patentes en un sector determinado. Deben entenderse, por tanto, como un punto de vista que motive una reflexión sobre las distintas facetas implicadas en el proceso de innovación. 


\section{Referencias}

Aho, E., Cornu, J., Georghiou, L. and Subirá, A. (2006). Creating an innovative Europe. Report of the independent expert group on $R \& D$ and innovation. European Communities, Belgium.

Álvarez, I., Fonfría, A. and Marín, R. (2005). The role of networking in the competitiveness profiles of Spanish firms, Working Paper 4, ICEI, UCM.

Arranz, N. and Fernández de Arroyabe, J.C. (2008). "The choice of partners in R\&D cooperation: An empirical analysis of Spanish firms". Technovation 28(1-2): 88-100.

Asociación Multisectorial de Empresas de Tecnologías de la Información, Comunicaciones y Electrónica (Asimelec) (2009). Informe 2009 del Sector TIC en España. Madrid: Asimelec.

Caballero, R. and Jaffe, A. (1993). "How high are the Giants' Shoulders: An Empirical Assessment of Knowledge Spillovers and Creative Destruction in a Model of Economic Growth". NBER Macroeconomics Annual, 8: 15-86.

Cassiman, B. (1999). "Cooperación en investigación y desarrollo: Evidencia para la industria manufacturera española". Papeles de economía española, 81: 143-154.

Castells, M. (1996). La era de la información: economía sociedad y cultura. Vol. I. La sociedad red. Madrid: Alianza Editorial.

Chesbrough, H. (2003). Open Innovation: The New Imperative for Creating and Profiting from Technology. Boston: Harvard Business School Press.

(2006). "Open Innovation: A New Paradigm for Understanding Industrial Innovation". En Chesbrough, H., Vanhaverbeke, W. and West, J. (Eds.). Open Innovation: Researching a New Paradigm. Oxford University Press, 1-12. 
Cotec (2000). "La innovación en las tecnologías de la información y las comunicaciones". Informes Cotec sobre el sistema español de innovación. Madrid: Fundación Cotec para la Innovación Tecnológica.

Dosi, G. and Malerba, F. (1996). "Organizational learning and institutional embeddedness". En Dosi, G.; Malerba, F. (Eds.), Organization and Strategy in the Evolution of Enterprise, Macmillan, London, 1-16.

Etzkowitz, H. and Leydesdorff, L. (1995). “The Triple Helix of University-Industry-Government Relations: A Laboratory for Knowledge Based Economic Development". EASST Review 14 (1): 11-19.

European Comission (2005). "Study on evaluating the knowledge economy - what are patents actually worth? The value of patents for today's economy and society". European Commission, Directorate-General for Internal Market. Tender MARKT/2004/09/E. Final Report.

Fabrizio, K. (2006). "The Use of University Research in Firm Innovation”. En Chesbrough, H.; Vanhaverbeke, W.; West, J. (Eds.). Open Innovation: Researching a New Paradigm. Oxford University Press. 134-160.

Freeman, Ch. (1995). "The National System of Innovation in Historical Perspective". Cambridge Journal of Economics, 19(1): 5-24.

García, E. (1992). "La cooperación interempresarial en España: Características de los acuerdos suscritos entre 1986 y 1989”. Economía Industrial, julioagosto, 113-122.

(1995). “Acuerdos de cooperación en I+D en España: un análisis empírico”. Revista Asturiana de Economía, 4: 195-207.

Georgescu-Roegen. N. [1971] (1999): The Entropy Law and the Economic Process, Universe, New York.

González, A. y De la Sota, D. (Coords.) (2006). La propiedad de la sociedad del conocimiento, Madrid. 
Griliches, Z. (1984). "Market Value, R\&D, and Patents”. En Griliches, Z. (Ed.) $R \& D$, Patents, and Productivity. University of Chicago Press, 249-252.

(1990). "Patent Statistics as Economic Indicators: A Survey", Journal of Economic Literature, 28(4): 1661-1707.

Guellec, D. and Van Pottelsberghe, B. (2000). “Applications, grants and the value of patent”. Economics Letters, 69(1): 109-114.

Gujarati, D. (2006). Principios de econometría. Madrid: McGraw Hill.

Hagedoorn, J. (1993). "Understanding the rationale of strategic technology partnering: inter-organizational modes of cooperation and sectoral differences". Strategic Managemen Journal, 14: 371-385.

Hall, B., Jaffe, A. and Trajtenberg, M. (2002). "The NBER Patent-Citations Data File: Lessons, Insights, and Methodological Tools". En Jaffe, A.; Trajtenberg, M. Patents, citations and innovations: a window on the knowledge economy. MIT, Boston, 403-460.

Handy, C. (1990). The age of unreason. Harvard Business School Press, Cambridge, Massachusetts. Tomado de Morcillo, P., 2006.

Hidalgo, A. (2003). Los patrones de innovación en España a través del análisis de patentes. Un análisis cualitativo del periodo 1988-1998. Madrid: Oficina Española de Patentes y Marcas.

Innometrics (2009). European Innovation Scoreboard 2008. Comparative analysis of innovation performance. UNU-MERIT.

Jacquemin, A., Lammerant, M. and Spinoy, B. (1986). Compétition Européenne et Cooperation Entre Entreprises en Matière de Recherche-Développement, Luxembourg : Office des Publications Officielles des Communautés Européennes.

Jaffe, A. (1986). "Technological Opportunity and Spillovers of R\&D: Evidence from Firms' Patents, Profits and market Value". American Economic Review, 76: 984-1001. 
Trajtenberg, M. and Henderson, R. (1993), "Geographic localization of knowledge spillovers as evidenced by patent citations". Quarterly Journal of Economics, 108: 577-598.

Leadbeater, C. (2006). Living on thin air. Harmondsworth: Penguin Books.

León, G. (2007). Evolución de las políticas de investigación e innovación de la Unión Europea y su impacto en España. Madrid: Universidad Politécnica de Madrid.

López, A. (2008). "Determinants for R\&D cooperation: Evidence from Spanish manufacturing firms". International Journal of Industrial Organization 26(1): 113-136.

Lundvall, B. (1992). National systems of innovation: towards a theory of innovation and interactive learning. London: Printer Publishers.

Maspons, R. and Escorsa, P. (2004). "Research networks. Flows of knowledge from and to cities: an analysis for Barcelona using patent statistics". Research evaluation, 13(2): 103-117.

Metcalfe, S. (1995). "The Economic Foundations of Technology Policy: Equilibrium and Evolutionary Perspectives". En Stoneman, P. (Ed.), Handbook of the Economics of Innovation and Technological Change, Oxford: Blackwell Publishers, 513-557.

Mintzberg, H. (1983). La naturaleza del trabajo directivo. Barcelona: Ariel.

Molero, J. e Hidalgo, A. (2003): "Los sectores de alta tecnología en la Comunidad de Madrid". En García, J. (Dir). Estructura económica de Madrid. Madrid: Civitas, 441-468.

Morcillo, P. (2006). Cultura e innovación empresarial. La conexión perfecta. Madrid: Thomson Editores.

Nelson, R. (1993). National Innovation Systems. A Comparative Analysis. New York: Oxford University Press. 
and Winter, S. (1977). "In search of a useful theory of innovation". Research Policy (6): 36-76.

(1982). An Evolutionary Model of Economic Change. The Belknap Press, Cambridge.

Niosi, J. (1993). "Strategic Partnerships in Canadian Advanced Materials". $R \& D$ Management, 23(1): 17-27.

Nonaka, I. and Takeuchi, H. (1995). The Knowledge Creating Company. New York: Oxford University Press.

Organisation for Economic Co-operation and Development (OECD) (1994). The measurement of scientific and technological activities: using patent dated as science and technology indicators. Patent Manual 1994. OECD.

(2005a). "Compendium of patent statistics 2005". OECD.

(2005b). Oslo Manual 3rd edition Guidelines for Collecting and Interpreting Innovation Data. OECD Publishing, Paris.

(2006). "The policy mix for research, development and innovation in Spain - Key issues and policy recommendations", DSTI/STP/TIP(2006)12/ FINAL.

Plaza, L. y Albert, A. (2004). "Análisis de la producción científica española citada en patentes biotecnológicas en EE.UU”. Revista española de documentación científica, 27(2): 212-220.

Powell, W., Koput, K., Smith-Doerr, L. and Owen-Smith, J. (1999). "Network Position and Firm Performance: Organizational Returns to Collaboration in the Biotechnology Industry". Research in the Sociology of Organizations. 16: $129-159$.

Rojo, R. y Gómez, I. (2006). “Análisis de la producción científica y tecnológica de la industria española en el sector de las Tecnologías de la Información y de las Comunicaciones". El profesional de la información. 15(3): 190-201. 
Rosenberg, N. (1982). Inside the Black Box: Technology and Economics. Cambridge New York: University Press.

Rozga, R. (1999). "Entre globalización tecnológica y contexto nacional y regional de la innovación, un aporte a la discusión de la importancia de lo global y lo local para la innovación tecnológica". V Seminario Internacional. Toluca, México.

Rubiralta, M. (2004). Transferencia a las empresas de la innovación universitaria. Descripción de modelos europeos. Madrid: Cotec.

Saxenian, A. (1994). Regional advance, culture and competition in Silicon Valley and Route 128, Harvard University Press, Cambridge, Mass.

Scherer, F. (1982). "Inter-industry technological flows in the United States". Research Policy, 11(4): 227-245.

Schmookler, J. (1962). "Economic Sources of Inventive Activity". Journal of Ecomomic History. XXII (1): 1-20.

Schumpeter, J. (1912). Theorie der wirtschaftlichen Entwicklung. Duncker\&Humblodt, Berlin. Versión en inglés, The Theory of Economic Development, Cambridge, Mass.: Harvard University Press, 1934. Citado de la versión en castellano, Teoría del desarrollo económico (1976), México: Fondo de Cultura Económica.

(1942). Capitalismo, socialismo y democracia. Trad. J. Díaz, Madrid: Aguilar.

Shapiro, C. and Varian, H. (1998). Information rules: a strategic guide to the network economy. Boston, Massachusetts: Harvard Business School Press.

Teece, D. (1989). "Interorganizational requirements of the innovation process". Managerial and Decision Economics. 10(1): 35-42.

Trajtenberg, M. (1990). "A Penny for Your Quotes: Patent Citations and the Value of Innovations”. RAND Journal of Economics, 21(1): 172-187. 
Verspagen, B. (2004). "Patents, Citations \& Innovations: A Window on the Knowledge Economy", Research Policy, 33: 1709-1711.

Villaseca, J. and Torrent, J. (2008). “TIC, conocimiento y crecimiento económico. Un análisis empírico, agregado e internacional, sobre las fuentes de la productividad". Economía Industrial, 360: 41-60.

Von Hippel, E. (1986). "Lead Users: A source of novel product concepts". Management Science, 32(7): 791-805.

West, J. and Gallager, S. (2006). "Open Innovation: A New Paradigm for Understanding Industrial Innovation". En Chesbrough, H.; Vanhaverbeke, W.; West, J. (Eds.). Open Innovation: Researching a New Paradigm. New York: Oxford University Press, 82-106.

World Intellectual Property Organization (WIPO) (2005). Exchanging value. Negotiating technology licensing agreements. Geneva: WIPO and International Trade Centre. 


\section{Anexo. Metodología de la investigación}

Considerando que el objetivo de este estudio es establecer un contraste sobre el modelo de innovación abierta en el sector de las TIC en España, a partir del análisis de patentes durante el periodo de 1999 al 2007, el primer paso fue el diseño de una base de datos propia.

a. Se incluyeron las solicitudes de patentes con número de prioridad española, lo que implica la extensión del colectivo a empresas extranjeras que protegen sus invenciones en España, bien por estrategia de protección o porque la invención ha sido realizada en el país.

b. Se seleccionaron aquellos registros donde al menos uno de los solicitantes de la patente fuese una empresa española.

c. Se han excluido las solicitudes presentadas por el propio inventor, dado que este trabajo de investigación se enfoca en empresas que hayan patentado sus innovaciones en cualquiera de los sectores que integran la industria de las TIC.

d. Se seleccionaron aquellas patentes publicadas, cuyo informe sobre el estado de la técnica ha sido favorable, lo que implica que no contienen antecedentes que afecten a la novedad o actividad inventiva. Por consiguiente, este colectivo se encuentra configurado por el conjunto de solicitudes de patentes que tienen un grado de calidad (Molero e Hidalgo, 2003).

e. Para efectuar la búsqueda, se utilizó la correspondencia de la Clasificación Internacional de Patentes (CIP), con base en los códigos del Compendio de Estadísticas sobre Patentes 2005 (OECD, 2005a). 
Cuadro A.1

Variables del modelo

\begin{tabular}{lc}
\hline Cooperación para la Innovación & (CoopInnov) \\
\hline Influencia de los Participantes & (PrtInf) \\
\hline Número de Inventores & INVNUM \\
Nacionalidad de los Inventores & INVNAT \\
\hline Búsqueda de Conocimiento Externo & (ExtSrc) \\
\hline Citas de patentes & PATDAT \\
Citas de artículos & CITDAT \\
\hline
\end{tabular}

